\title{
Photocatalytic Degradation of Organics by Using Nanocrystalline Titania
}

\author{
Sana Ahmad, ${ }^{1}$ Anam Nazir, ${ }^{1}$ and Tousif Hussain ${ }^{2}$ \\ ${ }^{1}$ Department of Chemistry, Lahore College for Women University, Lahore, Pakistan \\ ${ }^{2}$ Centre for Advanced Studies in Physics (CASP), GC University, Lahore, Pakistan \\ Correspondence should be addressed to Sana Ahmad; drsanaahmad@yahoo.com
}

Received 9 November 2017; Accepted 10 December 2017; Published 28 December 2017

Academic Editor: Mallikarjuna N. Nadagouda

Copyright (C) 2017 Sana Ahmad et al. This is an open access article distributed under the Creative Commons Attribution License, which permits unrestricted use, distribution, and reproduction in any medium, provided the original work is properly cited.

\begin{abstract}
Titanium dioxide nanoparticles were prepared by a modified sol-gel route. Titanium butoxide was used as precursor and nebulizer was used for a fine spray of particles. The prepared powders were characterized by FT-IR, SEM, XRD, and TGA-DTA methods. The results indicated that nanoparticles with small size and high surface area were synthesized. $\mathrm{XRD}$ result indicated that $\mathrm{TiO}_{2}$ nanoparticles were obtained in pure anatase crystalline form with a crystallite size of $40 \mathrm{~nm}$. The catalytic activity of as-synthesized nanoparticles was tested for the degradation of formic acid and toluene.
\end{abstract}

\section{Introduction}

The industrial growth has led to increase in the manufacture and growth of unused byproducts that cause severe environmental complications. Worldwide the researchers are working on several methodologies to overcome the issue. The use of light energy to waste mineralization is an important technique for the removal of industrial waste. $\mathrm{TiO}_{2}$ is extremely stable, of low cost, and safe for both living and nonliving things. Hence it is considered to be an almost perfect semiconductor for photocatalysis [1-3]. Various synthesis protocols of $\mathrm{TiO}_{2}$ nanoparticles have been reported in literatures that include hydrothermal method, solvothermal method, sol-gel route, precipitation method, and ultrasonic and microwave assisted methods. The sol-gel route is quite attractive for the preparation of nanosized $\mathrm{TiO}_{2}$ as it offers several advantages including high chemical purity, high homogeneity, purity, and flexibility in offering dopants in high concentrations, easy processing, control on composition, and stoichiometry $[4,5]$.

In the presence of UV or near UV irradiation, a wide range of organic compounds can be oxidized to $\mathrm{CO}_{2}$ and $\mathrm{H}_{2} \mathrm{O}$ on $\mathrm{TiO}_{2}$ catalyst surface at room temperature. The absorption of light results in the transfer of an electron from the valence band to the conduction band. This electron transfer process generates an electron-hole pair that imparts the redox properties to $\mathrm{TiO}_{2}$ surface. Many factors including the crystal nature and size of the photocatalyst affect the band gap value. Numerous researchers have reported the potential applications of $\mathrm{TiO}_{2}$ nanoparticles as a photocatalyst for the degradation of various organic compounds $[6,7]$.

In the present work, $\mathrm{TiO}_{2}$ nanoparticles were synthesized by a modified sol-gel route. Titanium butoxide was used as precursor and nebulizer was used for a fine spray of particles in an attempt to control particle size. The prepared powders were calcined at $550^{\circ} \mathrm{C}$ and characterized. The catalytic activity of as-synthesized nanoparticles was checked for the degradation of toluene and formic acid. Both formic acid and toluene were chosen as model compounds because they both degrade to simple degradation products like $\mathrm{CO}_{2}$ and $\mathrm{H}_{2} \mathrm{O}$. Toluene is an important indoor and industrial pollutant. It is aromatic and hence the efficiency of our prepared photocatalyst against an aliphatic (formic acid) and an aromatic organic species (toluene) was evaluated.

\section{Materials and Methods}

Titanium butoxide was purchased from Sigma Aldrich. Toluene, formic acid, $\mathrm{HCl}$, and butanol were purchased from $\mathrm{RDH}$. X-ray diffraction spectra of the powders were 
recorded at room temperature using a powder diffractometer (Jeol, Model: JDX-3523). Morphology determination was carried out with a scanning electron microscope (SEM), Jeol Jem-6480LV. The Fourier transform infrared spectrum (FTIR) was recorded on a Midac Corporation, Model: 2000. Ultraviolet-visible spectra were performed with a Hitachi Japan, U-2800 UV-Vis spectrophotometer while TGA analysis was performed with a NETZCH, STA-449C TGA-DTA instrument.

2.1. Preparation of Titanium Dioxide $\left(\mathrm{TiO}_{2}\right)$ Nanoparticles. A solution of titanium butoxide $(2 \mathrm{~mL}, 5.86 \mathrm{mmol})$ was prepared in butanol $(6 \mathrm{ml})$ in a conical flask. This solution was sprayed to a mixture of water and butanol $(3: 1)$ by using a nebulizer. The solution was left for stirring for 2 hours at room temperature. The $\mathrm{pH}$ of the solution was adjusted to 3 by using $\mathrm{HCl}(1 \mathrm{M})$. The solution was aged for $24 \mathrm{~h}$ at room temperature and a gel was formed. The gel was washed and dried in oven at $80^{\circ} \mathrm{C}$ for 10 hours. A white powder was obtained and was calcined at $550^{\circ} \mathrm{C}$ for 4 hours in air. A fine white powder consisting of $\mathrm{TiO}_{2}$ nanoparticles was obtained.

\subsection{Photocatalytic Activity}

2.2.1. Formic Acid Degradation. A small amount of $\mathrm{TiO}_{2}$ $(0.2 \mathrm{mg})$ was added in $0.1 \mathrm{M}$ formic acid solution $(200 \mathrm{ml})$ in a Pyrex glass beaker. The solution was held under sun light for 7 hours with occasional stirring. After certain time intervals, samples were taken out and were analyzed for total organic carbon (TOC) by titration.

2.2.2. Degradation of Toluene. In a conical flask, $100 \mathrm{ml}$ of $30 \mathrm{ppm}$ toluene solution was taken and $0.4 \mathrm{mg}$ of prepared $\mathrm{TiO}_{2}$ nanoparticles was added in the solution. The solution was stirred in dark for $20 \mathrm{~min}$ on a shaker and then exposed to sunlight for a certain period of time. Simultaneously, a blank experiment without the catalyst was carried out. At regular time intervals, a small amount of sample solution was taken out from the flask. UV-Vis absorption spectra were recorded to study the process of degradation.

In another experiment, degradation of toluene was studied under various $\mathrm{pH}$ values.

\section{Results and Discussion}

3.1. Characterization of the Photocatalyst. Figure 1 shows the FT-IR spectrum of the $\mathrm{TiO}_{2}$ nanoparticles calcined at $550^{\circ} \mathrm{C}$. An expected band between 500 and $800 \mathrm{~cm}^{-1}$ (Ti-O stretching) was seen which relates to the presence of Ti-O-Ti network. Two very small bands were observed at $3400 \mathrm{~cm}^{-1}$ and $1662 \mathrm{~cm}^{-1}$ that can be assigned to the stretching and bending vibration of $\mathrm{O}-\mathrm{H}$ group. This shows the existence of a small amount of moisture in the sample.

FT-IR result indicates that required $\mathrm{TiO}_{2}$ nanoparticles were successfully prepared which contain Ti-O-Ti network. No extra peak was observed which confirms the purity of our sample $[8,9]$.

TGA plot of the prepared $\mathrm{TiO}_{2}$ nanoparticles is given in Figure 2. The 1st mass loss of $2 \%$ was observed below



FIGURE 1: FT-IR spectrum of prepared $\mathrm{TiO}_{2}$ nanoparticles.

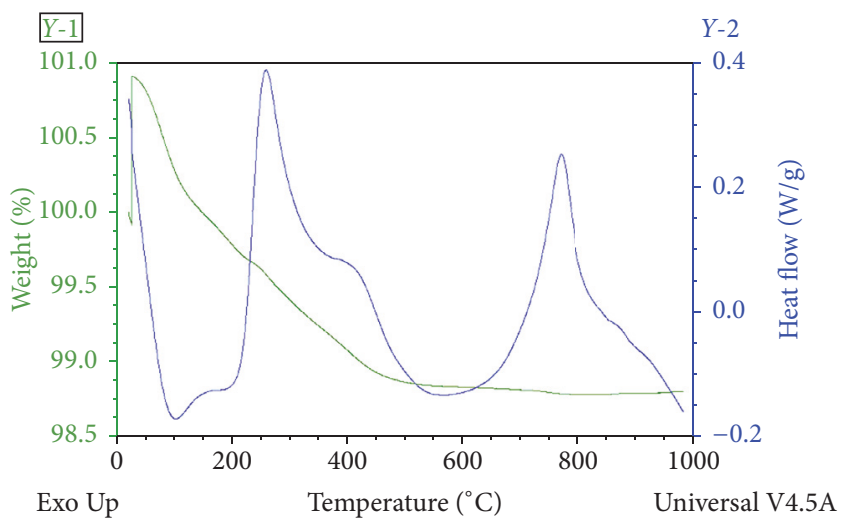

FIGURE 2: TGA plot of $\mathrm{TiO}_{2}$ nanoparticles.

$400^{\circ} \mathrm{C}$ and can be linked to either dehydroxylation of Ti-OH groups or to the removal of some moisture present in the sample. After that there is no further mass loss up to $1000^{\circ} \mathrm{C}$ which indicates good thermal stability of the prepared $\mathrm{TiO}_{2}$ nanoparticles at high temperature.

Figures 3(a) and 3(b) display SEM images of $\mathrm{TiO}_{2}$ nanoparticles. It is observed from the images that almost all nanoparticles are spherical. The particle size is approximately $50 \mathrm{~nm}$. Only a little particle aggregation was observed in the sample. No big clusters or rocks were seen in the images $[10,11]$.

Figure 4 shows the XRD patterns of $\mathrm{TiO}_{2}$ powder calcined at $550^{\circ} \mathrm{C}$. A typical diffractogram corresponding to anatase crystalline phase was obtained. It is noteworthy that no extra peaks corresponding to rutile or brookite phase were observed $[12,13]$.

Average crystallite size of the sample was calculated by using Scherrer's equation. The result confirms that only pure titanium dioxide nanoparticles were obtained in anatase crystalline form with average crystallite size of $40 \mathrm{~nm}$.

\subsection{Catalytic Activity}

3.2.1. Degradation of Formic Acid. Degradation rate of formic acid was estimated by measuring total organic carbon (TOC). TOC is calculated by simple titration method. After different 


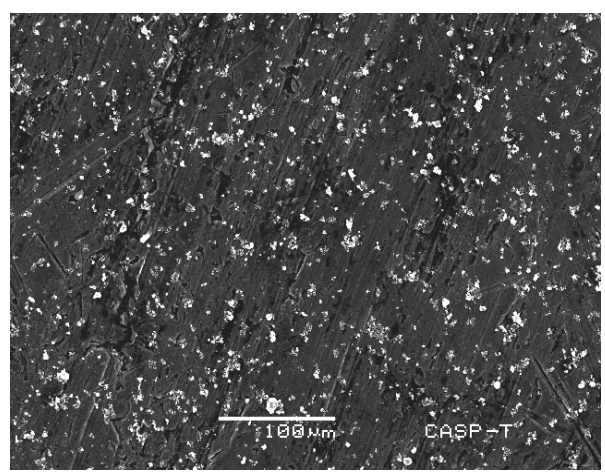

(a)

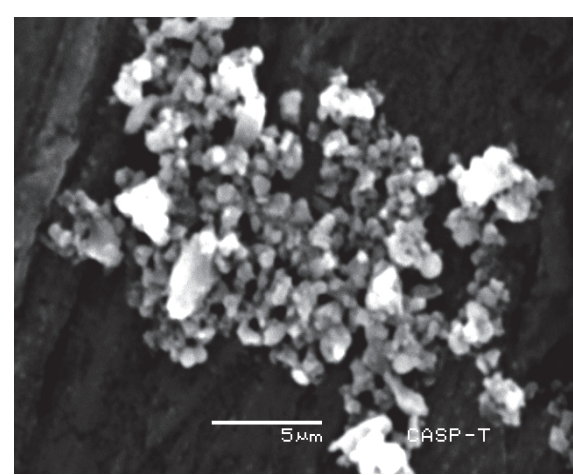

(b)

FIGURE 3: SEM images of prepared $\mathrm{TiO}_{2}$ nanoparticles.

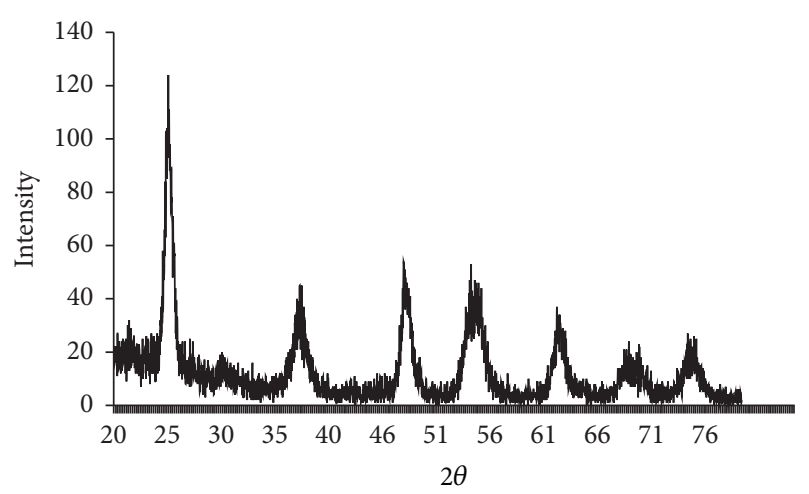

FIGURE 4: XRD pattern of $\mathrm{TiO}_{2}$ nanoparticles.

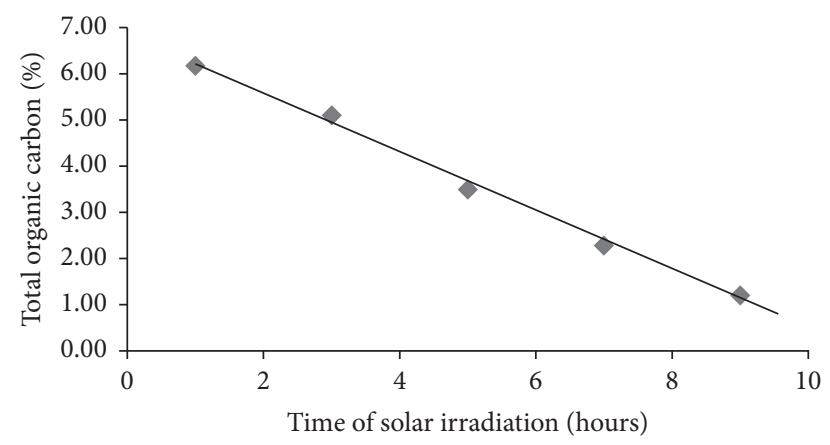

FIGURE 5: Degradation of formic acid under different times of solar irradiation at room temperature.

time intervals, the samples were taken from formic acid solution (under process of degradation in sunlight) and the process of titration was repeated.

TOC measurement indicates catalytic $\left(\mathrm{TiO}_{2} / \mathrm{UV}\right)$ degradation of formic acid under sunlight after 8 hours of the reaction. TOC was transformed from $6.17 \%$ to $1.20 \%$ (Figure 5) which relates to about $80 \%$ of formic acid removal in the experiment.

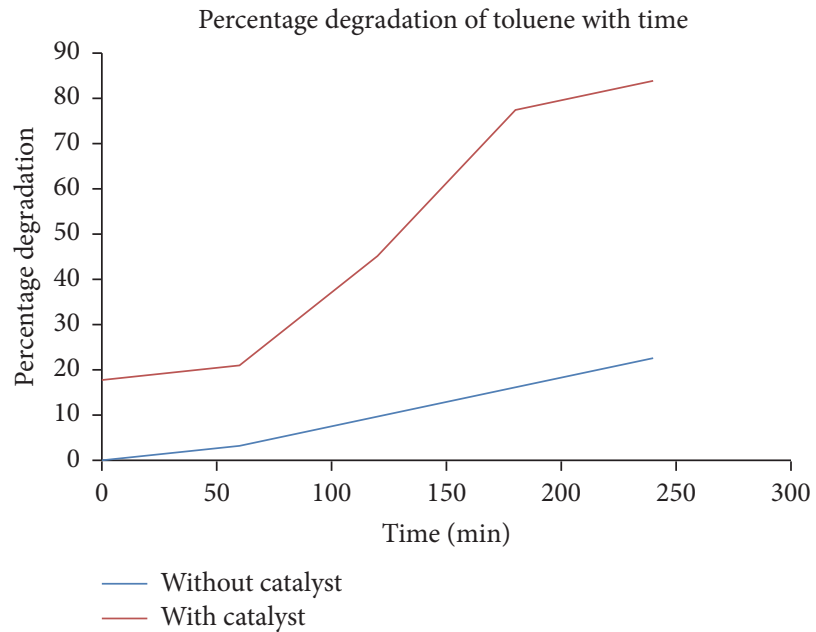

FIgURE 6: Degradation of toluene after different time intervals without and with $\mathrm{TiO}_{2}$ photocatalyst.

\subsubsection{Degradation of Toluene}

(1) Effect of Time on Photocatalytic Degradation of Toluene. The decrease in the absorbance at $244 \mathrm{~nm}$ wavelength, corresponding to the $\lambda$ max of toluene, was utilized to determine the extent of degradation of toluene. The degradation of toluene was checked after regular time intervals of 60 minutes. A blank experiment in the absence of $\mathrm{TiO}_{2}$ was also performed to study the effect of the photocatalyst. Results show that toluene was degraded rapidly in the presence of $\mathrm{TiO}_{2}$ photocatalyst on illumination with sunlight. In the absence of photocatalyst, however, the degradation was very slow as shown in Figure 6.

When a small amount of catalyst was added to the solution, it was noticed that the absorbance of the solution decreases, due to the adsorption of toluene molecules on the catalyst surface. When the solution was exposed to sunlight, a sharp decrease in the absorbance was observed indicating the degradation of toluene. The blank solution showed only a little change in absorbance indicating that the degradation of toluene mainly takes place due to the catalyst. 
TABLE 1: Pseudo-second-order kinetic model.

\begin{tabular}{lcccccc}
\hline \multirow{2}{*}{ Initial concentration } & & \multicolumn{2}{c}{ Pseudo-2nd-order } & \multicolumn{2}{c}{ With catalyst } \\
& $q e(\mathrm{~min} \cdot \mathrm{g} / \mathrm{mg})$ & $K_{2}(1 / \mathrm{min})$ & $R^{2}$ & $q e(\mathrm{~min} \cdot \mathrm{g} / \mathrm{mg})$ & $K_{2}(1 / \mathrm{min})$ & $R^{2}$ \\
\hline $30 \mathrm{ppm}$ & 29.440 & 7.990 & 0.9998 & 37.087 & 0.0367 & 0.9983 \\
\hline
\end{tabular}

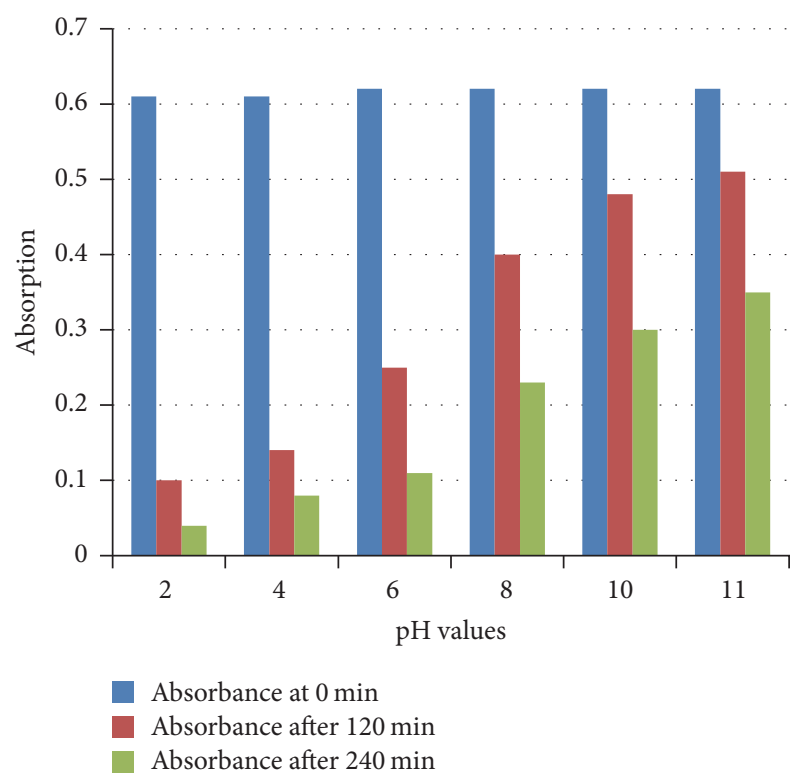

Figure 7: Degradation of toluene at various $\mathrm{pH}$ values.



FIgURE 8: Percentage degradation of toluene at various $\mathrm{pH}$ values.

The results show that the prepared $\mathrm{TiO}_{2}$ is an effective photocatalyst for the degradation of toluene. The toluene was almost completely degraded after 240 minutes of exposure of sunlight. The percentage degradation was about $83.8 \%$. In the absence of catalyst, the rate of degradation was very slow.

(2) Effect of $\mathrm{pH}$ on Degradation of Toluene. $\mathrm{pH}$ plays very important role in degradation process. Different toluene solutions were prepared having the same concentration (30 ppm) but with different $\mathrm{pH}$ values ranging from 2 to 11 . A small amount of catalyst $\left(\mathrm{TiO}_{2}\right.$ nanoparticles) was added in each solution and samples were exposed to sunlight for $240 \mathrm{~min}$. A marked decrease in toluene concentration was observed after $240 \mathrm{~min}$ in each sample. However, the maximum degradation was observed in acidic conditions at $2 \mathrm{pH}$ (Figures 7 and 8 ).

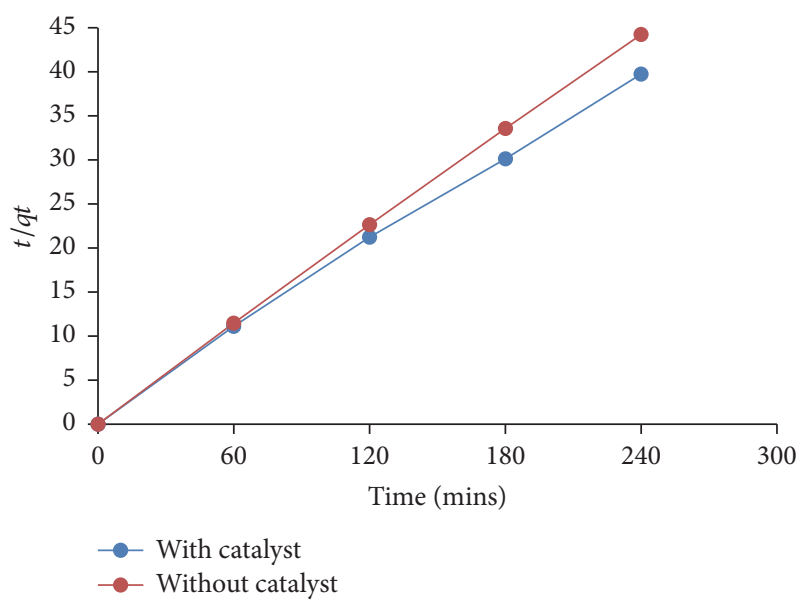

FIgUre 9: Pseudo-2nd-order kinetics.

In acidic $\mathrm{pH}$, electrostatic attraction is higher between protonated catalyst surface and the adsorbed species (toluene). In contrast, in basic $\mathrm{pH}$, the $\mathrm{TiO}_{2}$ becomes negatively charged which gives rise to a coulombic repulsion between the adsorbed species and the catalyst surface preventing the adsorption of the species. Hence the high photodegradation is observed in acidic $\mathrm{pH}$ and a marked decrease in the degradation is observed in basic $\mathrm{pH}$.

3.3. Kinetic Study. The kinetics for the degradation of toluene was studied by applying pseudo-first-order models. However, it was unsuccessful and then pseudo-second-order model was applied. The pseudo-second-order equation based on equilibrium adsorption is given as

$$
\frac{t}{q_{t}}=\frac{1}{k_{2} q_{e}^{2}}+\frac{t}{q_{e}}
$$

where $k_{2}(\mathrm{~g} / \mathrm{mg} \min )$ is the rate constant of second-order adsorption. A plot of $t / q_{t}$ versus time for different initial sorbent concentration was found to be linear for the degradation of toluene onto sorbent indicating the validity of equation and is given in Figure 9. The calculated $R^{2}, k_{2}$, and $q_{e}$ for pseudosecond-order kinetic model are shown in Table 1 . The experimental data showed a good compliance with the pseudosecond-order equation and the correlation coefficients for the linear plots were 0.99 for all the experimental data. These results suggested that the pseudo-second-order adsorption mechanism was predominant and that the overall rate for the degradation of toluene process appeared to be controlled by chemical process involving valence forces through sharing or exchange of electrons between sorbent and adsorbate. 


\section{Conclusion}

In summary, we have synthesized $\mathrm{TiO}_{2}$ nanoparticles by a modified sol-gel method. Titanium butoxide was used as precursor and a novel spray technique was used to hydrolyze the precursor. The particle size of prepared $\mathrm{TiO}_{2}$ nanoparticles was $40 \mathrm{~nm}$ with spherical morphology and anatase crystalline form. The catalytic activity of $\mathrm{TiO}_{2}$ nanoparticles for the degradation of formic acid and toluene was checked after different time intervals and variable $\mathrm{pH}$, under sunlight. Results concluded that the prepared $\mathrm{TiO}_{2}$ nanoparticles are effective catalyst for degradation of both formic acid and toluene. Kinetic study shows that the degradation of toluene follows pseudo-second-order reaction mechanism.

\section{Conflicts of Interest}

The authors declare that there are no conflicts of interest regarding the publication of this paper.

\section{References}

[1] Y. Yua, J. Wanga, and J. Parrb, "Preparation and properties of TiO2/fumed silica composite photocatalytic materials," Procedia Engineering, vol. 27, pp. 448-456, 2012.

[2] A. Shan, T. Ghazi, and S. Rashid, "Immobilisation of titanium dioxide onto supporting materials in heterogeneous photocatalysis: A review," Applied Catalysis, vol. 1, p. 389, 2010.

[3] M. Joshi, P. Mangrulkar, N. Labhsetwar, and S. Rayalu, "Silica gel supported titanium dioxide photocatalyst for methyl orange photoreduction," International Journal of Knowledge Engineering, vol. 3, pp. 137-139, 2012.

[4] U. Sajjad, A. Pasa, C. Alcantara, S. Bilmes, and U. RodriguesFilho, "Enhanced photocatalytic properties of core@shell SiO2@TiO2 nanoparticles," Applied Catalysis, B: Enviromental, vol. 179, pp. 333-343, 2015.

[5] W. Zhou, Y. Liu, B. Lei, and Y. Xu, "Preparation, characterization and photocatalytic activity of manganese doped $\mathrm{TiO} 2$ immobilized on silica gel," Journal of Hazardous Materials, vol. 160, pp. 78-82, 2008.

[6] Z. Tong, Q. Jiang, Z. Chen, Z. Jiang, and C. Li, "Two-step thermochemical cycles for $\mathrm{CO} 2$ splitting on $\mathrm{Zr}$-doped cobalt ferrite supported on silica," Solar Energy, vol. 116, pp. 133-143, 2015.

[7] T. Kaura, A. Srawa Wanchoob, and R. Wanchoob, "VisibleLight Induced Photocatalytic Degradation of Fungicide with Fe and Si Doped TiO2 Nanoparticles," Materials Today Proceedings, vol. 3, pp. 354-361, 2016.

[8] Y. Zhang and p. Li, "Porous $\mathrm{Zr}$-doped $\mathrm{SiO} 2$ shell/TiO2 core nanoparticles with expanded channels for photocatalysis," Materials and Design, vol. 88, pp. 1250-1259, 2015.

[9] M. Muneer, H. Abdul Amir, M. Abu Bakar, T. Mohd S, and S. Kamaruzzaman, "Synthesis and catalytic activity of $\mathrm{TiO} 2$ nanoparticles for photochemical oxidation of concentrated chlorophenols under direct solar radiation," International Journal of Electrochemical Science, vol. 7, pp. 4871-4888, 2012.

[10] B. Supat, W. Sumpun, S. Uraiwan, and P. Walailak, "Recyclable $\mathrm{UV}$ and visible light photocatalytically active amorphous $\mathrm{TiO} 2$ doped with M (III) ions ( $\mathrm{M}=\mathrm{Cr}$ and Fe)," Materials Chemistry and Physics, vol. 126, pp. 167-177, 2011.
[11] P. Posit and P. Sukon, "Titanium dioxide powder prepared by a sol-gel method," Journal of Ceramic Processing Research, vol. 10, pp. 167-170, 2009.

[12] L. Géraldine, M. Charline, M. Quentin, L. Stéphanie, D. Isabel, and $\mathrm{H}$. Benoît, "Doped sol-gel films vs. powders TiO2: On the positive effect induced by the presence of a substrate," Journal of Enviromental Chemical Engineering, vol. 4, pp. 449-459, 2016.

[13] R. Vijayalakshmi and K. Rajendran, "Effect of K+ Doping on the Phase Transformation of TiO2 Nanoparticles," AZojomo-Journal of Materials Online, vol. 6, pp. 1833-1843, 2010. 



Carbohydrate Chemistry

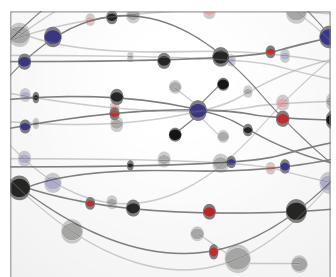

The Scientific World Journal
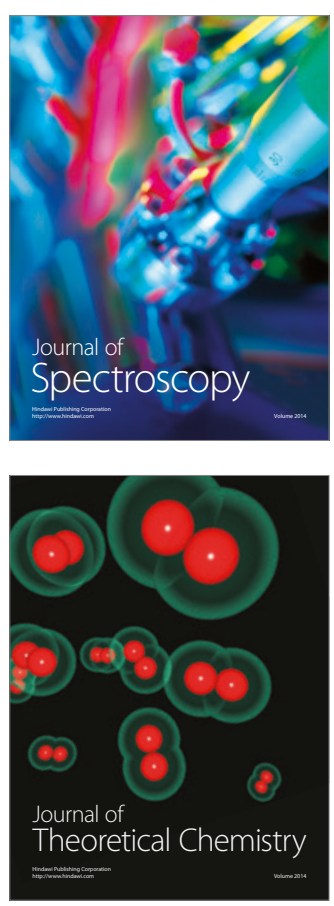
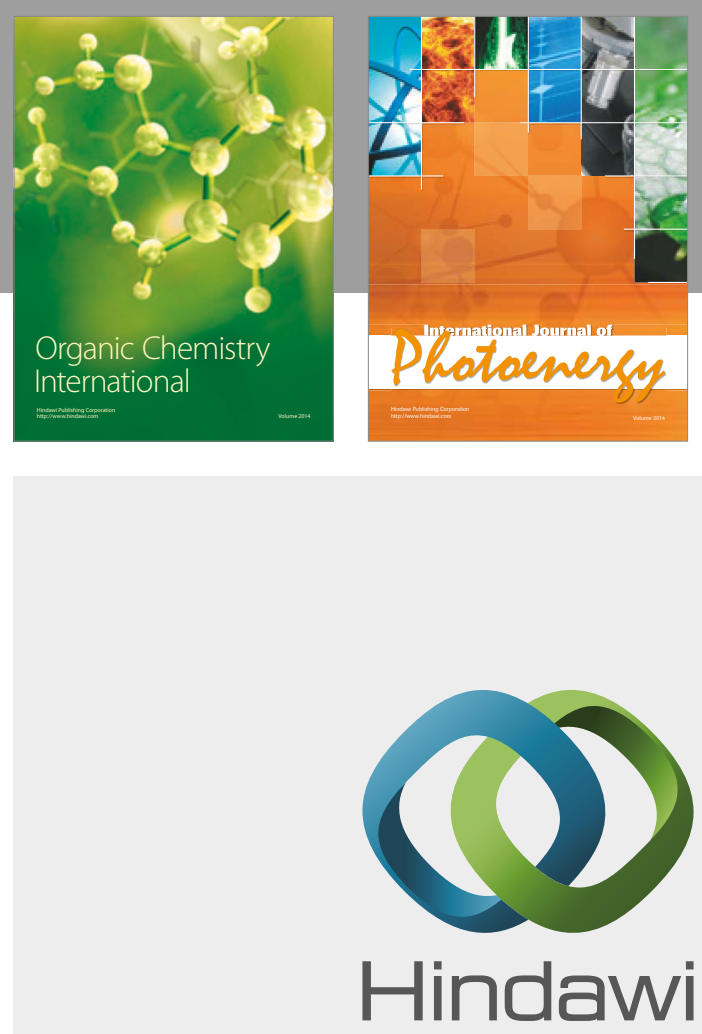

Submit your manuscripts at

https://www.hindawi.com

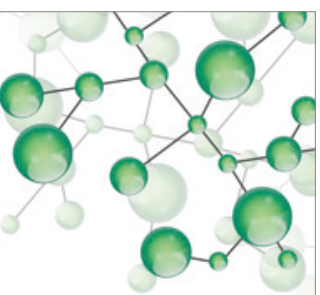

International Journal of

Inorganic Chemistry

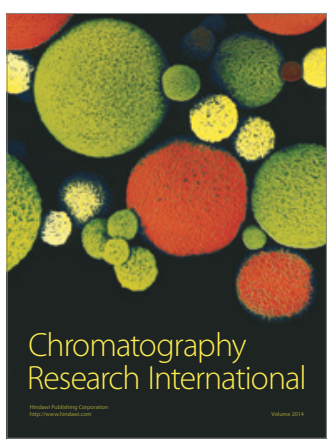

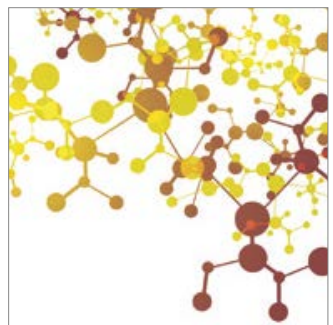

Applied Chemistry
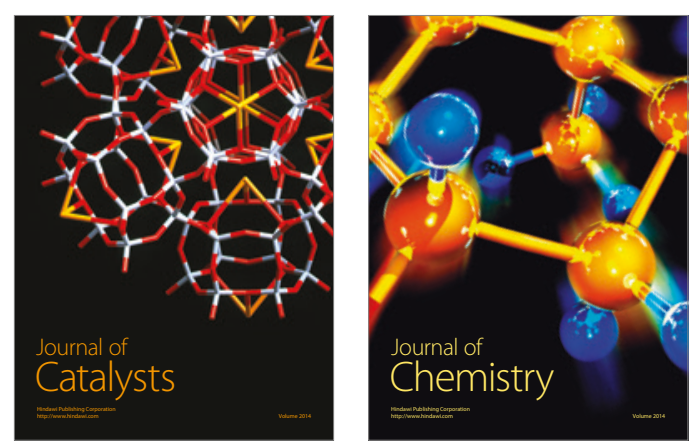
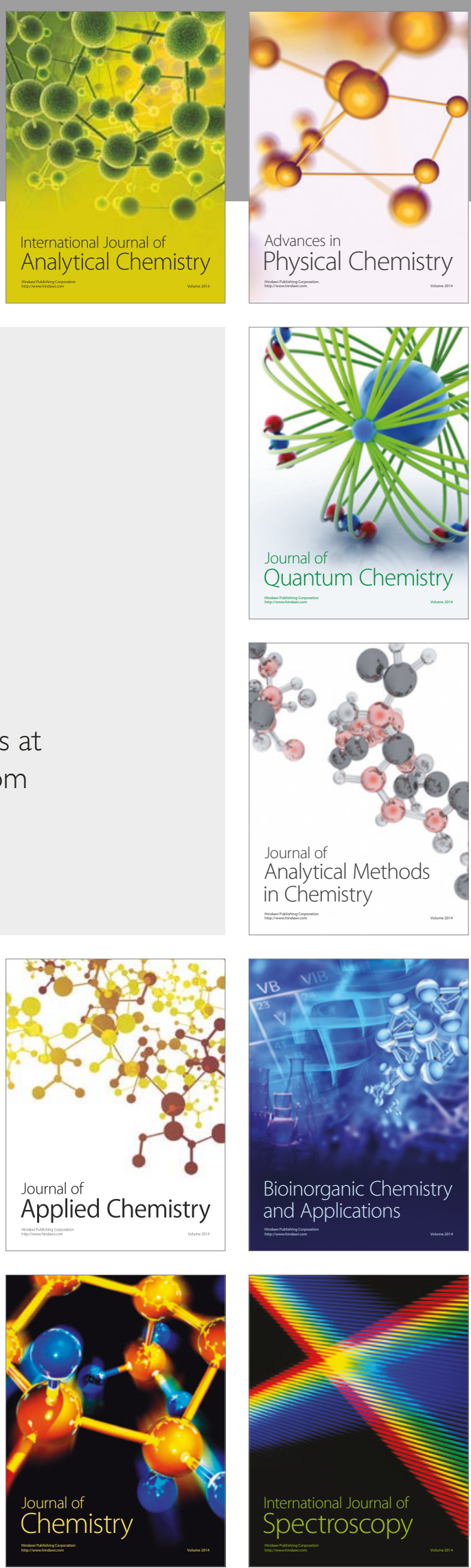